\title{
Simple Lyapunov Sampling for Event-Driven Control
}

\author{
Sylvain Durand* Nicolas Marchand ${ }^{* *}$ \\ Jose Fermi Guerrero Castellanos *** \\ * NeCS team, INRIA - GIPSA-lab - Univ. of Grenoble, Grenoble, \\ France (sylvain.durand@inrialpes.fr). \\ ** NeCS and SysCo teams, GIPSA-lab - CNRS - INRIA - Univ. of \\ Grenoble, Grenoble, France (nicolas.marchand@gipsa-lab.inpg.fr) \\ *** Autonomous University of Puebla (BUAP), Faculty of Electronics, \\ Puebla, Mexico (fguerrero@ece.buap.mx)
}

\begin{abstract}
In this paper, a simple Lyapunov sampling is proposed. Contrary to a periodic fashion which samples the system uniformly in time, an event-based scheme updates the control signal only when the system trajectory sufficiently changes. Furthermore, the present triggering mechanism is based on a Lyapunov function in order to enforce the events only when required from a stability point of view. Nevertheless, whereas the Lyapunov sampling mechanism initially introduced in Velasco et al. (2009) requires to execute a computationally heavy off-line algorithm, a fully on-line version is developed in this paper. The different approaches are tested (in simulation and practice) to show the efficiency of such an event-based control and, eventually, the performance remains ensured even if the constraint on the stability is relaxed.
\end{abstract}

Keywords: Event-based control, Lyapunov sampling, experimental tests

\section{INTRODUCTION}

The classical so-called discrete time framework of controlled systems consists in sampling the system uniformly in time with a constant sampling period $h_{n o m}$. This field is denoted the time-triggered case (or the synchronous case in sense that all the signal measurements are synchronous). Although periodicity simplifies the design and analysis, it results in a conservative usage of resources since the control law is computed and updated every time instants $t_{k}=k \cdot h_{n o m}$, that is at the same rate regardless it is really required or not. Actually, some works addressed more recently resource-aware implementations of the control law, using event-based sampling (also called asynchronous) where the control law is event-driven. Typical eventdetection mechanisms are functions on the variation of the state - or at least the output - of the system in order to update the control law when required from a performance point of view. This is the case in Årzén (1999); Sandee et al. (2005); Durand and Marchand (2009); Sánchez et al. (2009a); Heemels et al. (2009); Lunze and Lehmann (2010). In Åström and Bernhardsson (2002) in particular it is proved that such an approach reduces the number of sampling instants for the same final performance. An alternative approach also consists in enforcing events when required from a stability point of view, where the sampling is related to the variation of a Lyapunov function. This is notably depicted in Mazo et al. (2009); Anta and Tabuada (2010). Such a paradigm calls for resources whenever they are indeed necessary and, moreover, the stability of the controlled system is guaranteed even if the time between two samples is important. We then base our analysis more specifically on the Lyapunov sampling mechanism initially introduced in Velasco et al. (2009). This seminal work consists in enforcing the control updates only when the system trajectory reaches a given value, but relies on a heavy offline computation in order to find the sampling triggering condition. In this paper, we propose to lighten the algorithm in suggesting some relaxations of the Lyapunov sampling condition. This is applied to a state-feedback control law where the control parameter are deduced from the same Lyapunov function. The next section recalls the state-feedback background and more especially applies this architecture to even-driven systems. The Lyapunov sampling triggering mechanism is also introduced. The main contributions are then developed in section 2. At the end, our less-conservative proposals are successfully compared in simulation in section 3 and experimentally in section 4 (in terms of performance and computational needs).

\section{EVENT-DRIVEN STATE-FEEDBACK CONTROL BASED ON LYAPUNOV SAMPLING}

A linear continuous-time state-space representation is

$$
\begin{aligned}
& \dot{x}(t)=A x(t)+B u(t) \\
& y(t)=C x(t)
\end{aligned}
$$

and a state-feedback control law is given by

$$
u(t)=-K x(t)
$$

Take $\mathrm{P}$, a positive definite matrix solution of the Riccati equation $P A+A^{T} P-2 P B B^{T} P=-Q$, where $Q$ is also a positive definite matrix. Then,

$$
V(x)=x^{T}(t) P x(t)
$$


is a Lyapunov function which makes the system (1) stable if for all $x(t) \neq 0$ the control law (2) renders the Lyapunov derivative function strictly negative. A common solution is choosing $K=B^{T} P$ and the control law finally becomes

$$
u(t)=-B^{T} P x(t)
$$

Eventually, an event-driven control scheme updates the control signal only at some discrete instants. Let $t_{a}$ be the beginning time of the current control sample, that is the last time an event occurs, and $t_{a+1}$ be the next time a control signal will be calculated. The sampling interval $h(\cdot)$ hence depends on these time instants, i.e. $h\left(t_{a}\right)=t_{a}-t_{a-1}$ for instance. Furthermore, the control signal is constant during the whole sampling interval, which yields

$$
\forall t \in\left[t_{a}, t_{a+1}\right) \quad u(t)=-K x\left(t_{a}\right)=-K x_{a}
$$

In the present study case, the sampling instants are enforced with respect to $V(x)$. Indeed, an event condition based on Lyapunov functions was investigated in Velasco et al. (2009). Considering that constant values of a Lyapunov function define some contour curves that form closed regions around the equilibrium point, the proposed sampling mechanism enforces job executions each time the system trajectory reaches a given contour curve. Such a sampling mechanism is illustrated in Fig. 1 for a two-state system. Actually, the discretization of a given Lyapunov function in the energy space domain - that is the $\left(x_{1}, x_{2}\right)$ plane in this case - defines a set of ellipses of constant energy. The system trajectory can then move between them without requiring control actions and control jobs are only activated each time the trajectory intersects a contour curve from outside to inside. Therefore, by construction, the generated samples are stable in the Lyapunov sense since the system energy decreases event after event. This triggering mechanism is called Lyapunov sampling.

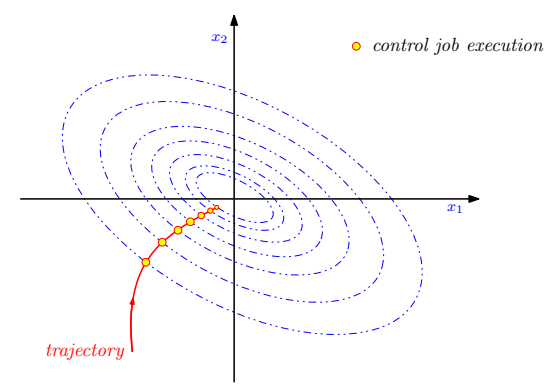

Fig. 1. Lyapunov sampling principle for a two-state system

\subsection{Lyapunov sampling}

The Lyapunov sampling mechanism is enforced when

$$
V\left(x_{a}\right)=\eta V\left(x_{a-1}\right)
$$

where the energy gain factor $\eta$ is a tunable parameter used for event detection: for some small values of $\eta$ large sampling periods are expected whereas large values will reduce the time between two events. By construction, the sampling scheme is stable in the discrete Lyapunov sense if $\eta$ is restricted to $0<\eta<1$.

\subsection{Stable Lyapunov sampling}

Although the Lyapunov sampling guarantees stable sampling sequences in the Lyapunov sense, nothing is ensured about the stability of the continuous-time dynamics. Indeed, if the system energy increases before the next sampling instant, the condition from (4) will not be validated and an event will hence not occur. Two Lyapunov sampling triggering cases are hence possible. If $\eta$ is correctly chosen the system trajectory will cross contour curves again and again until achieving the equilibrium point. On the other hand, with a non adapted $\eta$ value the system energy could increase before achieving the next ellipse. In this case, a new event will never occur and the system becomes unstable. For this reason, an infinite sequence of samples has to be generated. One has to determine when the energy decrease produced by the system trajectory up to the point it starts is gaining energy again and, then, placing a contour curve passing for that point would guarantee a new sample. Actually, this strongly relates to the energy gain factor $\eta$ and, therefore, a restriction in the Lyapunov sampling condition is required. Finally, an infinite sampling sequence is ensured if

$$
\eta^{*}<\eta<1
$$

where $0 \leq \eta^{*} \leq 1$ is the minimum energy gain factor. This condition presents the stable Lyapunov sampling mechanism.

\subsection{Event-detection improvement}

As already explained, the Lyapunov sampling mechanism allows to activate some control jobs only when the trajectory intersects a contour curve from outside to inside. A restriction is then added in order to guarantee that contour curves are crossed again and again and so is decreasing the system energy. However, if the next curve could not be achieved for a certain reason (such as if a perturbation occurs) the system trajectory will diverge anyway - going from inside to outside - without (almost) any chance to cross again that contour curve. For this reason, we propose to add a safety condition in the event-detection scheme in order to enforce a job execution as soon as the system energy increases, that is when

$$
\begin{aligned}
& \Delta V>0 \\
& \text { with } \Delta V=V\left(x_{a}\right)-V\left(x_{a-1}\right)
\end{aligned}
$$

\section{A LESS-CONSERVATIVE LYAPUNOV SAMPLING}

The idea is to soften the sampling scheme introduced in Velasco et al. (2009) and depicted above. Actually, the stable Lyapunov sampling mechanism is based on restricting $\eta$ in the Lyapunov sampling condition (4), in such a way that the energy decreases again and again at each sample. The resulting restriction is given in (5). However, computing the minimum energy gain factor is not trivial because this is a non-convex problem. Indeed, $\eta^{*}$ is calculated for a given system to control and a given Lyapunov function. Moreover, whereas some simplifications exist for linear systems, this parameter cannot be easily calculated for nonlinear systems and, finally, its existence is not 
(always) guaranteed. As a result, the resulting algorithm is computationally heavy and will probably take a long time, this is why it needs to be executed off line. Therefore, we propose to relax the constraint on the energy gain factor (in order to not have to use $\eta^{*}$ in the algorithm anymore). On the other hand, once obtained the minimum energy gain factor (when existing) a "stable" value - just greater than $\eta^{*}$ - is then applied in the sampling condition (4) for the whole running time. This is highly conservative since a large value will reduce the time between events. This value can also be large insomuch - very closed to 1 - as some events will occur (quasi)-continuously. Eventually, $\eta^{*}$ is obtained in calculating the minimum achieved energy without changing the control signal for any initial condition $x_{0}$ (assuming that this minimum exists), that is

$$
V^{*}\left(x_{0}\right)=\min _{t} V\left(x\left(t, x_{0}\right)\right) \quad \forall t \geq 0
$$

where $x\left(t, x_{0}\right)$ is the solution of the closed-loop system (1) when $u(t)=-K x_{0}$. The value that minimizes the minimum distance between the initial energy $V\left(x_{0}\right)$ and the minimum achieved energy $V^{*}\left(x_{0}\right)$ for all possible initial conditions then gives the minimum energy gain factor

$$
\eta^{*}=\max _{x_{0}} \frac{V^{*}\left(x_{0}\right)}{V\left(x_{0}\right)}
$$

One could refer to Velasco et al. (2009) for further details. Consequently, it can be assumed that the dynamics of the system is stable even for a large number of values of $\eta$ smaller than $\eta^{*}$. This is why we also propose to make dynamically varying the energy gain factor. This will allow to enlarge the sampling intervals when the system is stable. One just has to keep in mind that the system could become unstable and, therefore, quickly react when this appends. Let

$$
\eta\left(t_{a}\right)=\eta_{a}
$$

be the varying energy gain factor at the current sampling instant $t_{a}$. This parameter is now updated in the eventdriven fashion and then used in the Lyapunov sampling condition (4), which becomes

$$
V\left(x_{a}\right)=\eta_{a-1} V\left(x_{a-1}\right)
$$

where $0<\eta(\cdot)<1$, by construction yet.

Relaxation 1: The first guess is to slowly decrease $\eta$ when the energy decreases (the constraint on the energy gain factor is relaxed when the system is stable in order to distance the events) and changing it back to $\eta^{*}$ as soon as the energy increases (in order to stabilize the system again since this minimum value ensure a stable sampling sequence). The resulting algorithm is

$$
\eta_{a}= \begin{cases}\eta^{*}+\varepsilon & \text { if } \Delta V>0 \\ (1-\underline{\nu}) \eta_{a-1} & \text { otherwise }\end{cases}
$$

where $\varepsilon \in \mathbb{R}^{+}$allows to guarantee the left inequality in (5) while $0<\underline{\nu}<1$ leads to decrease the value of the energy gain factor. Note that $\Delta V$ was already defined in (6). Anyway, this first relaxation still requires the heavy offline algorithm to calculate $\eta^{*}$.

Relaxation 2: An on-line version can be easily obtained replacing this parameter by the right inequality limit in the sampling condition (5) (since this value also ensure a stable sequence). This yields

$$
\eta_{a}= \begin{cases}1-\varepsilon & \text { if } \Delta V>0 \\ (1-\underline{\nu}) \eta_{a-1} & \text { otherwise }\end{cases}
$$

The paying tradeoff is an increase of the sampling instants, because the system (almost) runs with a time-triggered behavior as soon as the system becomes unstable, that is when $\eta(t)=1-\varepsilon$. Nevertheless, the cost of the resulting fully on-line algorithm is strongly cut.

Relaxation 3: Such as we previously suggested to slowly decrease the energy gain factor when the system is stable, we now propose to slowly increase it while the system dynamics is unstable. The idea is that the system becomes stable with a value lower than $\eta^{*}$ for some initial conditions (as explained in introduction). Eventually, in the case where the system is still not stabilized, the value of $\eta(\cdot)$ is increased again and again until achieving a stable value. This leads

$$
\eta_{a}= \begin{cases}(1+\bar{\nu}) \eta_{a-1} & \text { if } \Delta V>0 \\ (1-\underline{\nu}) \eta_{a-1} & \text { otherwise }\end{cases}
$$

where $\bar{\nu}>0$ while a saturation function is required to not overshoot the maximum possible value $1-\varepsilon$.

Relaxation 4: Actually, the latter expression can be reformulated. Firstly, the energy gain factor is function of the variation of the Lyapunov function, which can hence be used to decide when increase or decrease its value. This leads to

$$
\eta_{a}=(1+v \Delta V) \eta_{a-1}
$$

where $v \in \mathbb{R}^{+}$is a tunable parameter. Moreover, one could note that $\Delta V$ is already calculated in the Lyapunov sampling mechanism improvement (6), which means that the computational cost of this algorithm is not increased. Then, it is also possible to take into account the sampling interval in order to highly correct the energy gain factor when the system becomes unstable after a large steadystate time. The resulting relation is

$$
\eta_{a}=\left(1+v h\left(t_{a}\right) \Delta V\right) \eta_{a-1}
$$

One could note that, in fact, the sampling interval naturally comes from the discretization of $\dot{\eta}(t)=v \dot{V}(x) \eta(t)$ (using the backward difference approximation).

\section{SIMULATION RESULTS: APPLICATION TO A DOUBLE INTEGRATOR SYSTEM}

In order to compare our proposal with the original work of Velasco et al. (2009), we propose to simulate the same system in Matlab/Simulink. This is the double integrator system, whose state-space representation (1) is given by

$$
A=\left[\begin{array}{ll}
0 & 1 \\
0 & 0
\end{array}\right] \quad \text { and } \quad B=\left[\begin{array}{l}
0 \\
1
\end{array}\right]
$$

The initial condition is

$$
x_{0}=\left[\begin{array}{c}
0 \\
-3
\end{array}\right]
$$

and the control parameters in (2) and (3) are 


$$
P=\left[\begin{array}{cc}
1.1455 & 0.1 \\
0.1 & 0.0545
\end{array}\right] \text { and } K=\left[\begin{array}{ll}
10 & 11
\end{array}\right]
$$

The bench used to test the different strategies is a $10 \mathrm{~s}$ simulation done with Matlab/Simulink.

The Lyapunov sampling mechanism consists in enforcing a control job when the system trajectory crosses a given level, such as defined in subsection 1.1. This scheme is firstly simulated for two different values of the energy gain factor whose results are shown in Fig. 2. The left plot shows the system trajectory in the energy space domain - that is the $\left(x_{1}, x_{2}\right)$ plane in the present case - while the right side represents the system energy in the top plot, i.e. the Lyapunov function, and the evolution of the sampling intervals with respect to time in the bottom one. The total number of samples is also indicated.

- In Fig. 2(a) with $\eta=0.8$, the system is stable in the Lyapunov sense (since the energy function decreases) and the dynamics of the continuous system is stable too (since the trajectory system tends to the origin). Moreover, the performances are quite similar to the classical approach - not represented here - with a real reduction of the number of samples (about $91 \%$ less than with a periodic sampling period $h_{n o m}=0.01 s$ ).

- In Fig. 2(b) with $\eta=0.65$, the system is unstable. Indeed, the system diverges after a certain time because the next level is never achieved due to a bad value of the energy gain factor. Thus, the triggering condition is never satisfied and, as a result, the control signal is not updated anymore.

Fortunately, the stable Lyapunov sampling mechanism allows to avoid that problem in restricting the energy gain factor. Nevertheless, a simple solution - introduced in subsection 1.3 - consists in calculating the control signal as soon as the system energy increases, in order to be reactive when such an unstable behavior occurs. The resulting simulation results are shown in Fig. 3, where the sampling intervals vary more chaotically but the system trajectory finally goes to the origin. Furthermore, this solution is interesting for some systems which have to track a given setpoint, since the Lyapunov function will inevitably increase each time the setpoint changes. For this
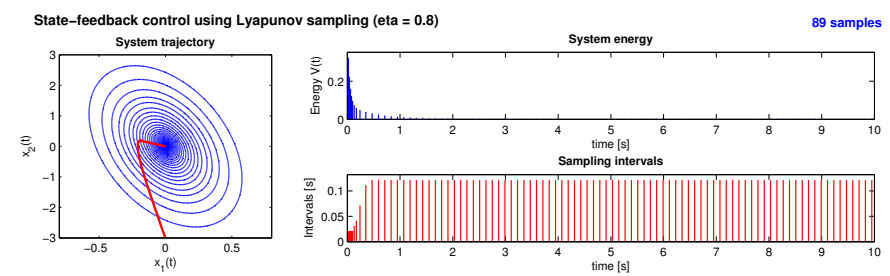

(a) Stable Lyapunov sampling with $\eta=0.8$
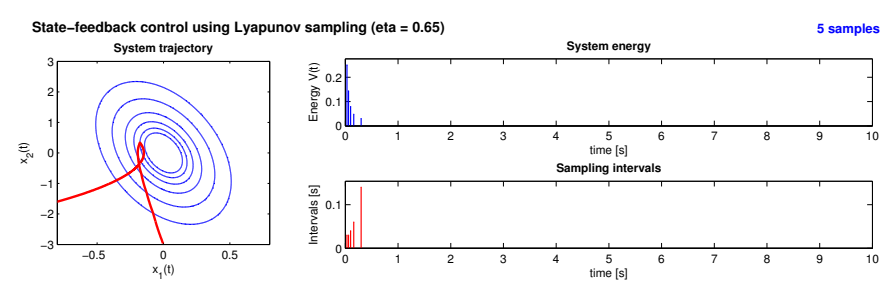

(b) Unstable Lyapunov sampling with $\eta=0.65$

Fig. 2. Lyapunov sampling: stable and unstable behaviors. reason, the event detection improvement is applied in the following simulations.

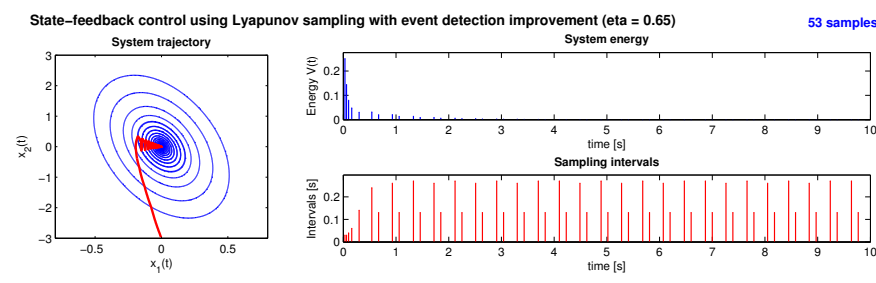

Fig. 3. Lyapunov sampling with event-detection improvement.

A stable Lyapunov sampling mechanism - detailed in subsection 1.2 - is required to guarantee that the system energy always decreases and the next level is hence achieved. This is related to the energy gain factor which has to be restrained in such a way that the generated sequence of samples is infinite, where the minimum energy gain factor $\eta^{*}$ bounds the restriction. Applying the suggested algorithm to the present study case finally yields $\eta^{*}=0.7818$, like highlighted in Fig. 4. The latter figure plots the ratio between $i$ ) the minimum achieved energy needed to compute the Lyapunov function from the initial condition until it is gaining energy again and $\boldsymbol{i i}$ ) the initial energy, for any initial condition. See Velasco et al. (2009) for further details. Then, applying $\eta^{*}<\eta<1$ guarantees a stable sampling sequence. One could verify, and besides, that both stable and unstable behaviors illustrated in Fig. 2 verify this condition (the system is unstable using the first value and stable in the second case). However, whereas the algorithm allows a stable sequence, it is computationally heavy and has to be executed off-line (it takes few seconds to dozens of seconds to calculate $\eta^{*}$ ).

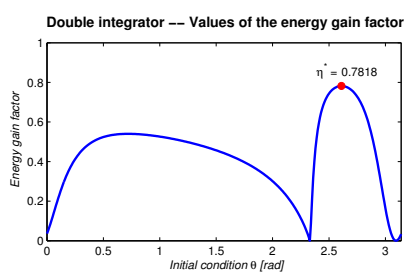

Fig. 4. Results of the off-line algorithm to calculate $\eta^{*}$ in the double integrator study case.

The main contribution in this paper is to 1) relax the constraint on the energy gain factor in order to lighten the algorithm and 2) make this parameter dynamically varying because the system is stable in the Lyapunov sense for a large number of values of $\eta$ smaller than $\eta^{*}$ (as one can see in Fig. 4). The different strategies are detailed in section 2 and the corresponding simulation results are represented in Fig. 5. Note that the bottom right plot now represents the dynamics of $\eta(t)$.

Relaxation 1: The first proposal simply consists in decreasing $\eta$ when the system is stable and going back to the stable value $\eta^{*}$ as soon as it becomes unstable. This intuitive guess leads to run with a value less than $\eta^{*}$ during about $90 \%$ of the simulation time - as one can see in Fig. 5(a) - decreasing again the number of samples (almost $50 \%$ less than with the original stable Lyapunov sampling) with still good performances. 
Relaxation 2: The previous relaxation is then modified in order to not use $\eta^{*}$ anymore but the greater stable limit instead, in order to have a fully on-line algorithm. The results - in Fig. 5(b) - are quite close to the previous ones with a number of samples which is naturally increased.

Relaxation 3: Whereas the previous algorithms applied a stable value when the system becomes unstable, the energy gain factor is now slowly increased. The results are represented in Fig. 5(c) where one could see a smoother variation of $\eta$.

Relaxation 4: The last algorithm is another formulation of the previous one. The simulation results are drawn in Fig. 5(d) where the dynamics of the energy gain factor is clearly toned down.

The value of the different parameters needed in the above algorithms and used for these simulation results are $\bar{\nu}=$ $\underline{\nu}=0.05, \varepsilon=0.02$ and $v=6.2$.
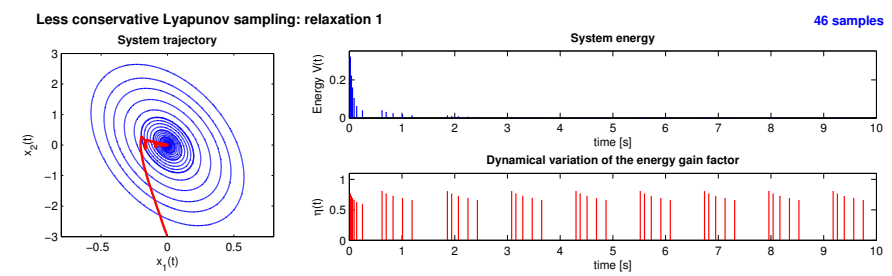

(a) Relaxation 1
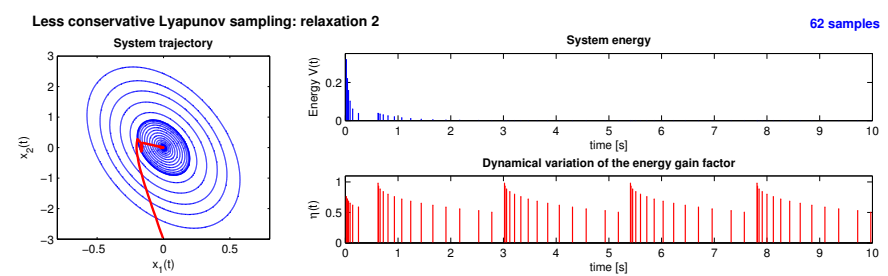

(b) Relaxation 2
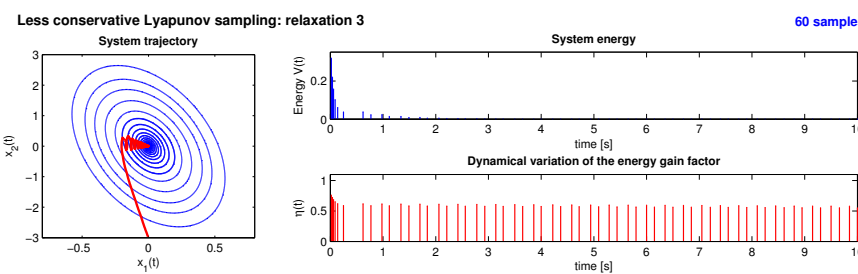

(c) Relaxation 3
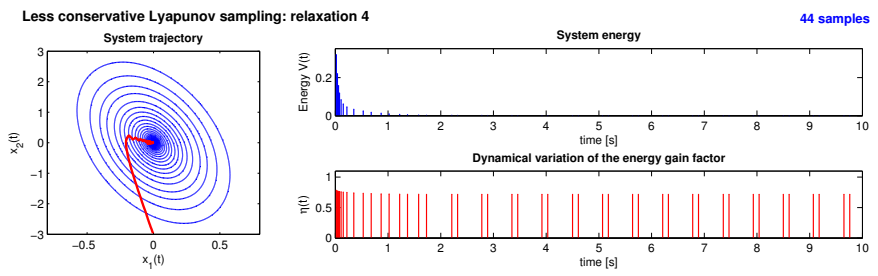

(d) Relaxation 4

Fig. 5. Less-conservative Lyapunov sampling.

At the end, one has to keep in mind that, even if the system trajectory is not as "direct" as the classical time-triggered or the original stable Lyapunov sampling methods, our proposals have advantages on both techniques: $i)$ the number of samples is highly reduced (94\% of samples less than with the classical way and $50 \%$ less than the Lyapunov sampling) and $\boldsymbol{i i}$ ) the less-conservative strategies are ex- ecuted in real-time and do not require a computationally heavy off-line algorithm. Furthermore, the last relaxation eventually allows a system response very close to the existing techniques, as one can see in Fig. 6 when looking at the dynamics of the system.

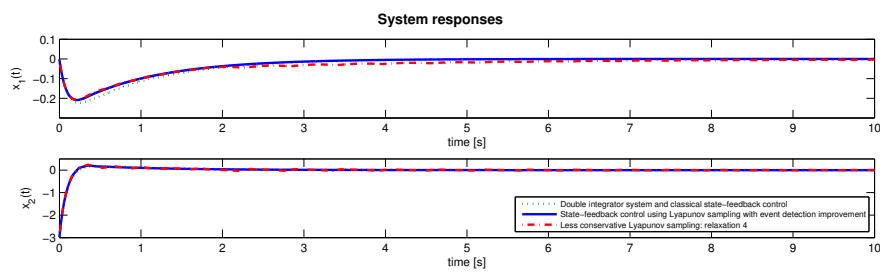

Fig. 6. System response: comparison of the classical sampling, the stable Lyapunov sampling and the lessconservative proposal (with relaxation 4).

\section{EXPERIMENTAL RESULTS: CONTROL OF THE POSITION OF A MOTOR}

A practical DC servo-motor provides the control force to a cart (through a belt drive system). A digital controller then acts on the motor and a potentiometer allows to measure the position from its rotation. Note that the system runs in real-time in the Matlab/Simulink environment. Let

$$
\begin{aligned}
& \dot{x}(t)=A \cdot x(t)+B \cdot u(t) \\
& y(t)=C \cdot x(t)
\end{aligned}
$$

be the state-space representation of the system to control. In the present case, the output is the position and the control signal is the voltage which power supplies the electric motor. A simple identification leads to

$$
A=\left[\begin{array}{cc}
-16.67 & 0 \\
1 & 0
\end{array}\right], \quad B=\left[\begin{array}{c}
72.03 \\
0
\end{array}\right], \quad C=\left[\begin{array}{ll}
0 & 1
\end{array}\right]
$$

The state-feedback strategy then consists in multiplying the states $x$ of the system by a certain gain and setting this product as the new system input, as introduced in (2). Furthermore, as the system will track a given reference $r(t)$, an extra term is added in the control law. This finally yields $u(t)=-K x(t)+K_{r} r(t)$, where $K_{r}$ is a tunable gain defined by

$$
K_{r}=-\left(C(A-B K)^{-1} B\right)^{-1}
$$

An integral state $\dot{z}(t)=r(t)-y(t)$ is also added in order to ensure a null steady-state error. The control law becomes

$$
u(t)=-K x(t)+K_{r} r(t)+K_{z} z(t)
$$

where $K_{z}$ is a new tunable parameter. The resulting augmented closed-loop system thus yields

$$
\left[\begin{array}{c}
\dot{x}(t) \\
\dot{z}(t)
\end{array}\right]=\left[\begin{array}{cc}
A-B K & B K_{z} \\
-C & 0
\end{array}\right]\left[\begin{array}{c}
x(t) \\
z(t)
\end{array}\right]+\left[\begin{array}{c}
B K_{r} \\
1
\end{array}\right] r(t)
$$

Whereas the integral term $z$ is required to control the system it does not need to be applied in the eventdetection mechanism. This means the Lyapunov function remains $V(x)=x^{T} P x$ like in (3). Eventually, the control parameters are $K=\left[\begin{array}{ll}0 & 0.25\end{array}\right], K_{r}=0.25, K_{z}=1$ and 


$$
P=\left[\begin{array}{cc}
2.9406 & 0 \\
0 & 0.2747
\end{array}\right]
$$

The time-triggered sampling interval is $h_{n o m}=0.01 \mathrm{~s}$ while the parameters required in the asynchronous strategies are $\bar{\nu}=\underline{\nu}=0.1, \varepsilon=0.02$ and $v=1$.

Some performances indexes are indicated in the following experimental results, that are the number of samples required to perform the test bench and some other ones initially introduced in Sánchez et al. (2009b):

- The IAE index gives information on the setpoint tracking

$$
I A E=\int_{0}^{\infty}|e(t)| d t
$$

- The IAEP index compares the time-based and eventbased system responses

$$
I A E P=\int_{0}^{\infty}\left|y_{\text {time-based }}(t)-y_{\text {event-based }}(t)\right| d t
$$

- The IAD index compares the time-based and eventbased IAE

$$
I A D=\int_{0}^{\infty}\left|I A E_{\text {time-based }}(t)-I A E_{\text {event-based }}(t)\right| d t
$$

The classical approach is represented in Fig. 7, where the top plot shows the setpoint and the measurement whereas the bottom one shows the system energy, that is the Lyapunov function.

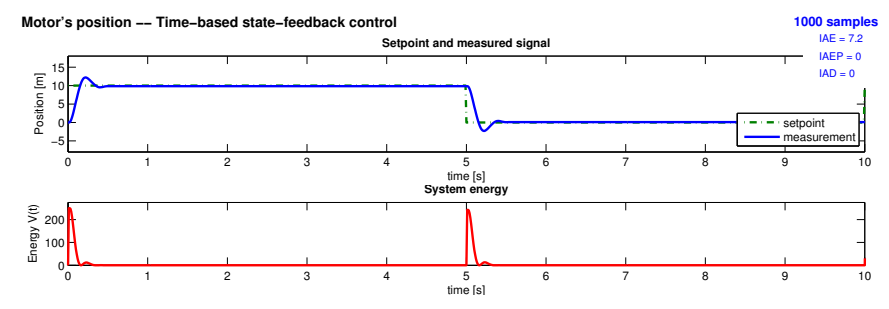

Fig. 7. Time-triggered state-feedback control.

The stable Lyapunov sampling mechanism enforces a control update only when required from a stability point of view while promising an infinite sequence of samples, therefore ensuring the stability of the system dynamics. This is detailed in subsection 1.2. However, this requires to calculate the energy gain factor for any initial condition, such as depicted in Fig. 8, in order to then deduce the minimum energy gain factor $\eta^{*}$. In the present study case, this latter parameter does not exist since the obtained

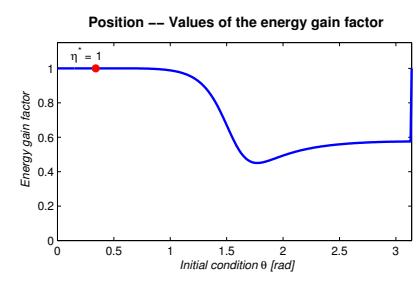

Fig. 8. Results of the off-line algorithm to calculate $\eta^{*}$ in the position's control study case. value $\eta^{*}=1$ could not satisfy the restricting condition (5), and so is the stability. Consequently, the original Lyapunov sampling mechanism cannot be applied to control the position of the cart. An issue is hence required.

Firstly, an event-detection improvement (6) was proposed in subsection 1.3. It consists in reacting as soon as the system becomes unstable. As a result, applying this small improvement now allows to run the system whenever the value of the energy gain factor in the sampling mechanism (4). This leads to the simulations results in Fig. 9 with $\eta=0.1$ for instance. In fact, this small value allows to considerably reduce the number of samples but the system becomes unstable sometimes. Whatever, the proposed improvement leads to stabilize it back.

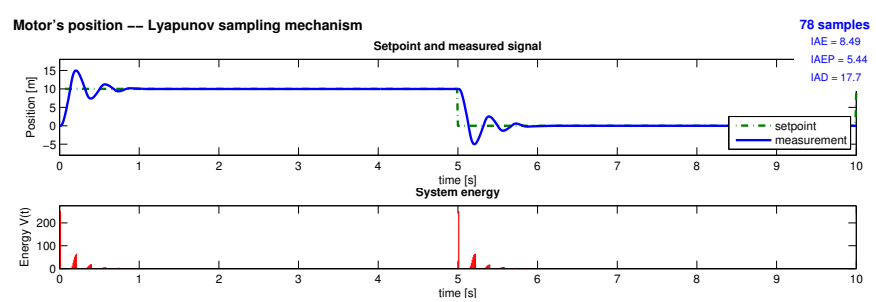

Fig. 9. Lyapunov sampling with event-detection improvement.

Another solution was suggested in section 2 to not have to calculate $\eta^{*}$ and, consequently, not run the computationally heavy off-line algorithm. The energy gain factor is now varying and dynamically changes in order to increase the time between two events when the system is stable and, respectively, decrease it back when the system becomes unstable. The experimental results of the less-conservative algorithms are drawn in Fig. 10. An extra plot represents the dynamics of $\eta(t)$. Note that all the experiments lead to an important reduction of computation since only (less than) $10 \%$ of samples allow to achieve almost the same performance (the IAEP index for instance - which compares the event-based system response with the timetriggered one - is low in all cases).

Relaxation 1: The first relaxation is not tested since it still needs $\eta^{*}$.

Relaxation 2: The second approach consists in slowly decreasing the energy gain factor when the Lyapunov function decreases and drastically going to a (quasi) time-triggered sampling as soon as the system becomes unstable, without requiring $\eta^{*}$ anymore. The results are drawn in Fig. 10(a), where the different performance indexes are all very low.

Relaxation 3: The third proposal slowly decreases / increases $\eta(t)$. The results are represented in Fig. 10(b) and lead to reduce the number of samples again.

Relaxation 4: Another formulation results in Fig. 10(c), where the system response is the closest to the classical one (looking at the indexes of performances).

Eventually, some perturbations are enforced during the running time of the experiments in order to see if an eventbased scheme correctly reacts when the system does not work as well as in theory. The test bench is extended to a $20 s$-experiment with two steps and the drive-shaft of the motor is sometimes slowed down (as one can see in Fig. 4 at 3 and $12 \mathrm{~s}$ ). The system is robust to this perturbation 

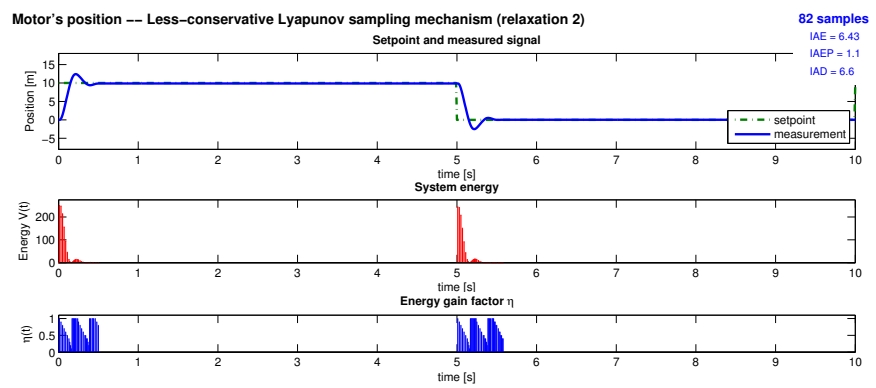

(a) Relaxation 2
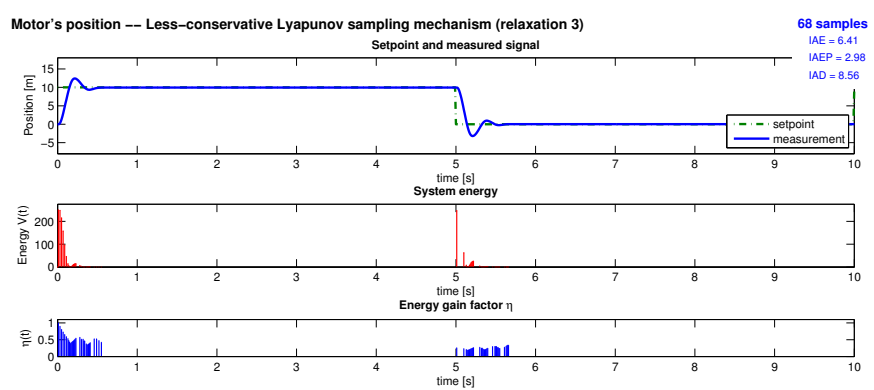

(b) Relaxation 3
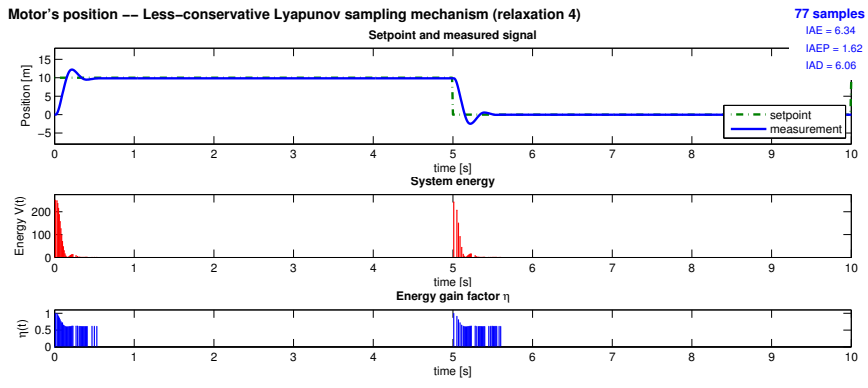

(c) Relaxation 4

Fig. 10. Less-conservative Lyapunov sampling.

since the position continues to track the given setpoint. This is because the Lyapunov function changes during the perturbations and so are enforced new events. As a result, the number of samples increases to dynamically take into account the perturbation.

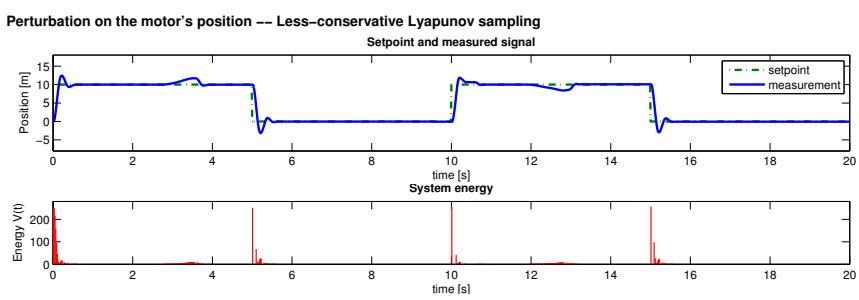

Fig. 11. Robustness to some perturbations in the less conservative Lyapunov sampling case.

\section{CONCLUSIONS AND FUTURE WORKS}

This paper contributes to a simple Lyapunov sampling. The principle consists in updating the control signal only when required from a stability point of view, that is when a Lyapunov function reaches a given energy level. Whereas the Lyapunov sampling mechanism initially developed in Velasco et al. (2009) is based on restricting the Lyapunov sampling condition and requires to execute a computationally heavy off-line algorithm, we propose in this paper to relax this constraint. This eventually yields a fully on-line version and the performance remains unchanged, even if the system is not sampled during a long time. A reduction of more than $90 \%$ of samples less than in the time-triggered principle and $50 \%$ less than in the original mechanism is achieved when controlling a double integrator in simulation. Furthermore, our proposal can be applied to control any system, which was not the case before. About $90 \%$ of samples less than in the classical approach is achieved when experimentally controlling the position of an electric motor, while the original Lyapunov sampling mechanism was not directly applicable to the tested system. The advantage of an asynchronous scheme is hence highly highlighted and the encouraging results strongly motivate to continue developing event-based control strategies.

\section{REFERENCES}

Anta, A. and Tabuada, P. (2010). To sample or not to sample: Self-triggered control for nonlinear systems. IEEE Transactions on Automatic Control, 55, 2030 2042.

Årzén, K.E. (1999). A simple event-based PID controller. In Preprints of the 14th World Congress of IFAC. Beijing, P.R. China.

Åström, K. and Bernhardsson, B. (2002). Comparison of Riemann and Lebesque sampling for first order stochastic systems. In Proceedings of the 41st IEEE Conference on Decision and Control.

Durand, S. and Marchand, N. (2009). Further results on event-based PID controller. In Proceedings of the European Control Conference.

Heemels, W., Sandee, J., and van den Bosch, P. (2009). Analysis of event-driven controllers for linear systems. International journal of control, 81, 571-590.

Lunze, J. and Lehmann, D. (2010). A state-feedback approach to event-based control. Automatica, 46, 211215.

Mazo, M.J., Anta, A., and Tabuada, P. (2009). On selftriggered control for linear systems: Guarantees and complexity. In Proceedings of the 10th European Control Conference.

Sánchez, J., Guarnes, M., and Dormido, S. (2009a). On the application of different event-based sampling strategies to the control of a simple industrial process. Sensors, 9 , 6795-6818.

Sánchez, J., Guarnes, M., Dormido, S., and Visioli, A. (2009b). Comparative study of event-based control strategies: An experimental approach on a simple tank. In Proceedings of the 10th European Control Conference.

Sandee, J., Heemels, W., and van den Bosch, P. (2005). Event-driven control as an opportunity in the multidisciplinary development of embedded controllers. In Proceedings of American Control Conference, 1776-1781.

Velasco, M., Martí, P., and Bini, E. (2009). On lyapunov sampling for event-driven controllers. In Proceedings of the 48th IEEE Conference on Decision and Control. 